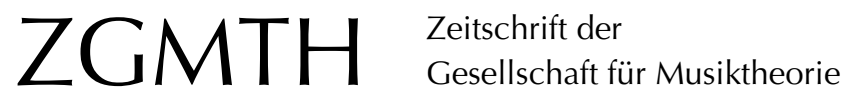

Bosi, Carlo (2016): Astrid Opitz, Modus in den Chansons von Binchois (= Saarbrücker Studien zur Musikwissenschaft, Bd. 18), Sinzig: Studio 2015. ZGMTH 13/1, 151-155. https://doi.org/10.31751/884

(C) 2016 Carlo Bosi

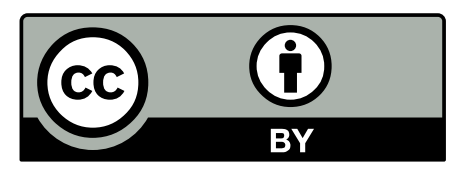

Dieser Text erscheint im Open Access und ist lizenziert unter einer Creative Commons Namensnennung 4.0 International Lizenz.

This is an open access article licensed under a Creative Commons Attribution 4.0 International License.

eingereicht / submitted: 03/01/2017 angenommen / accepted: 10/01/2017 veröffentlicht / first published: 20/02/2017 zuletzt geändert / last updated: 10/05/2018 


\section{Astrid Opitz, Modus in den Chansons von Binchois (= Saarbrücker Studien zur Musikwissenschaft, Bd. 18), Sinzig: Studio 2015}

Wenngleich das Thema des Modus in Bezug auf das mehrstimmige weltliche Repertoire des späten Mittelalters und der frühen Renaissance in den letzten Jahrzehnten, sowohl vonseiten seiner Befürworter als auch seiner Gegner, allmählich an Aufmerksamkeit gewonnen hat', sind Monografien eher selten, besonders wenn sie sich auf das Werk einzelner Komponisten beschränken. ${ }^{2}$ Umso willkommener ist daher diese Dissertation von Astrid Opitz, welche in die von Rainer Kleinertz geleitete Reihe Saarbrücker Studien zur Musikwissenschaft aufgenommen wurde. Die ausschließliche Fokussierung auf das Chansonwerk von Gilles Binchois ist deshalb bemerkenswert, weil dieser erstens neben Guillaume Dufay als einer der wichtigsten und einflussreichsten Chansonkomponisten des ausgehenden Mittelalters gilt, zweitens weil die Melodieführungen vieler seiner Chansons einen ganz anderen als den tatsächlichen Schlussklang erwarten lassen, was Dennis Slavin als »Binchois game« kennzeichnet. ${ }^{3}$ Und genau dieses Binchois-Spiel gab den Anstoß für Opitz' Arbeit, wie die Autorin selbst in der Einleitung ausführt (13).

Nach der kurzen Einleitung teilt sich das Buch thematisch in zwei große Abschnitte: Am Anfang stehen drei Kapitel über allgemeine theoretische und musikhistorische Ansätze zu Modalität und Mehrstimmigkeit, hieran schließen drei weitere Kapitel über die Chansons von Binchois an, wobei in jedem dieser Kapitel ein Modus im Zentrum steht. Wie sie

1 Vgl. Meier 1953; Treitler 1965; Berger 1992; Powers 1992; Perkins 1996; Strohm 1996; Fuller 1998; Bosi 2013.

2 Vgl. Bosi 2013.

3 Vgl. Slavin 2000. schon in der Einleitung klar darlegt, vertritt die Autorin die These, "dass Binchois' Chansons (wie auch die seiner Zeitgenossen) stark modal geprägt sind « (17) und dass sich ein Modus anhand bestimmter Merkmale »in der Melodie- und Satzgestaltung" (18) erkennen lasse. Im darauffolgenden ersten Kapitel setzt sich die Autorin mit Methoden auseinander, die in der Forschung bisher zur Analyse weltlicher Mehrstimmigkeit im Allgemeinen und der Chansons Binchois' im Besonderen angewendet wurden. Dass sie funktionsharmonische Ansätze als ungeeignet betrachtet, ist bereits aus der Kürze des ihnen gewidmeten Kapitelabschnitts abzuleiten. Und in der Tat verwirft sie klar und deutlich die ahistorische Einstellung vieler analytischer Ansätze, die standardisierte, auf Dreiklangakkorden beruhende Kadenzformeln in einem Repertoire zu orten versuchen, in dem sie noch nicht in einem modernen Sinne vorhanden waren, weil der Kern der sharmonischen Fortschreitung aus dem zweistimmigen Gerüstsatz cantus/tenor bestand. Unter den modalen Theorien unterscheidet Opitz solche, die sich an der Hexachordlehre orientieren, von den sogenannten spseudo-klassischen und solchen, die auf gregorianischen Formeln basieren. Während die erste Kategorie vor allem auf eine Hypothese von Gaston Allaire und deren praktische Anwendung auf die weltliche Musik des 14. und des frühen 15. Jahrhunderts durch Christian Berger zurückgeht ${ }^{4}$, gründet sich die spseudo-klassisches modale Theorie auf die Lehre von den Quarten- und Quintenspezies, deren wichtigste Vertreter italienische Theoretiker wie z. B. Marchettus von Padua waren und die ausführlich von Johannes Tinctoris in seinem

$4 \quad$ Vgl. Allaire 1972; Berger 1992. 
Liber de natura et proprietate tonorum (1476) erörtert wird. ${ }^{5}$ Unter den verschiedenen modernen Verfechtern dieser Theorie bezieht sich die Autorin auf Aufsätze von Leo Treitler, Reinhard Strohm und vor allem Carlo Bosi. ${ }^{6}$ Dass sie die Lehre von den Spezies zur Bestimmung des Modus als ungeeignet erachtet, geht aus ihrem Text deutlich hervor, der die melodiebildenden und -gestaltenden Potenziale der Spezies hinterfragt. Eher neigt Opitz der Herangehensweise von Bernhard Meier zu, der neben den Spezies ausdrücklich Formeln aus der Gregorianik herbeizieht. ${ }^{7}$ Und tatsächlich bringt Opitz die von Meier bloß entworfene Methode im Laufe ihrer Monografie zu ihrer vollen Entfaltung, wie weiter unten verdeutlicht wird. Schließlich nimmt die Autorin weitere, sowohl von funktionalharmonischen als auch von modalen Lehren abweichende Ansätze unter die Lupe wie die kontextbezogene Theorie eines spitch of referencer nach Thomas Brothers ${ }^{8}$, Schenker-ähnliche Prolongationsanalysen und die Theorie der stonal color von Graeme Boone ${ }^{9}$ (Letzterer geht von einer im Vorhinein bestimmten und durch chromatische Färbungen nuancierten einheitlichen `Tonalität ‘ aus, die im Prinzip der Schlusston, unabhängig von Akzidentiensetzung und lokalen modalen Gewichtsverlagerungen, offenbart). In den zwei weiteren Kapiteln des ersten Teils ihrer Arbeit fasst Opitz die wichtigsten mittelalterlichen Theorien zur Modalität in der Ein- und Mehrstimmigkeit zusammen, wobei verständlicherweise der Mehrstimmigkeit viel mehr Aufmerksamkeit eingeräumt wird. Dabei ignoriert sie außer einer knappen Passage aus dem Jacobus von Lüttich zugeschriebenen Compendium de musica (um 1300) die wenigen indirekten vor dem Berkeley Manuskript (um 1375) auftretenden Belege für modale Mehrstimmigkeit. Meine in diesem Sinne vor-

\footnotetext{
5 Vgl. Tinctoris 1975.

6 Vgl. Treitler 1965; Strohm 1996; Bosi 2013.

7 Vgl. Meier 1953.

8 Brothers 1991.

9 Boone 1997.
}

genommene Interpretation einer Textstelle aus dem Lucidarium in arte musicae planae des Marchettus von Padua (ca. 1318) ${ }^{10}$ kritisiert sie aufgrund eines Missverständnisses. Bei meiner Deutung des Marchettus-Zitats "Sed quia quilibet cantus, sive sit perfectus sive imperfectus etcetera, de aliquo modorum existit $[\ldots] \ll^{11}$ hatte ich mich nicht, wie Opitz meint, auf »etcetera" bezogen, um auf eine implizierte Anwendbarkeit der Modalität auf polyphone Sätze hinzudeuten, sondern auf »quilibet cantus«, denn der Begriff >cantus` kann zu dieser Zeit sowohl den scantus planusı, d.h. die monophone gregorianische Tradition der westlichen Kirche, als auch den 'cantus mensuratus` bezeichnen - ein Begriff, der im Allgemeinen auf Mehrstimmigkeit hinweist. $^{12}$

Im Grunde genommen versucht Opitz zu belegen, dass einer mehrstimmigen Chanson - und im Allgemeinen einem mehrstimmigen Satz - im Wesentlichen ein einziger Modus zuzuordnen sei und dass dessen Merkmale am besten durch der gregorianischen Tradition entnommene Formeln veranschaulicht werden. Dass Tinctoris unter den von Opitz angeführten Theoretikern den größten Raum beansprucht, ist kein Wunder, denn er ist in der Tat der einzige Autor vor Pietro Aaron, der praktische Beispiele zur Erläuterung seiner Moduslehre anbietet (die meisten davon sind allerdings einstimmig). Auch bei den Analysen der Chansons von Binchois, die den zweiten Teil des Buchs bilden, stellen die sexempla s aus Tinctoris' Liber das Gros der für jeden Modus als charakteristisch herangezogenen Formeln: So leitet eine längere Auswahl solcher Formeln jedes der drei den Chansons gewidmeten Kapitel ein. Drei diesbezügliche

10 Vgl. Bosi 2007, 25.

11 »Aber weil jede Art von Gesang, sei er perfekt oder imperfekt usw. in einem gewissen Modus ist/existiert [...]«. Vgl. Marchettus von Padua 1987, 368 (10.1.7 und 10.1.8) (Übersetzung des Verfassers).

12 In dieser Hinsicht ist die Übersetzung Jan W. Herlingers (auf den Opitz sich bezieht) von »cantus« als »melody« irreführend; vgl. ebd. 


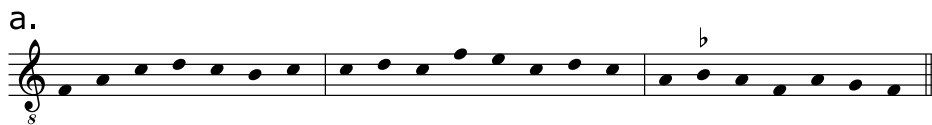

b.

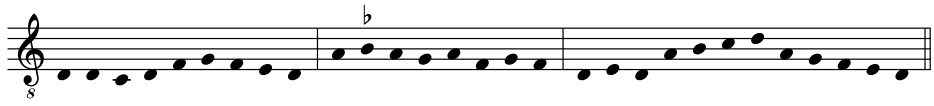

c.

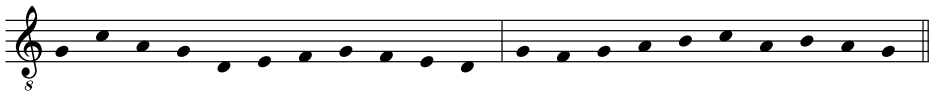

Beispiel 1: Johannes Tinctoris, Liber de natura et proprietate tonorum (1476), Exempla: a. ex. 18 [e] (»Exemplum quinti toni «) $)^{13}$; b. ex. 18 [a] (»Exemplum primi toni «) ${ }^{14}$; c. ex. 63 [h] (»Exemplum octavi «) $)^{15}$

Beispiele aus Tinctoris' Liber, die auch Opitz in ihrem Buch verwendet, sollen dies vor Augen führen (Bsp. 1).

Dabei übersieht die Autorin, dass die Beispiele von Tinctoris bereits rationalisierte, ad hoc komponierte Modelle bilden, die idealerweise einer optimierten Schilderung bzw. Visualisierung der Spezies dienen sollen, wenngleich manchmal gregorianische ‘Fragmentes aufzutauchen scheinen. Das ist deshalb problematisch, weil Opitz so in den meisten Fällen den Beispielen Tinctoris' einen authentischen sgregorianischen Charakter beimisst.

Weil der Autorin zufolge fast jede Chanson in einem bestimmten Modus komponiert ist und weil in den Chansons von Binchois, wie in vielen seiner Zeitgenossen, die maneria des deuterus, wenn überhaupt, nur als flüchtige Andeutung vorkommt, sind die letzten drei Kapitel, den restlichen maneriae des tritus, protus und tetrardus gewidmet, ihrerseits nach authentischen und plagalen Modi untergliedert. Für jeden authentischen bzw. plagalen Modus wählt Opitz eine Chanson aus, die die jeweilige Modalität exemplarisch darstellen soll, in der also die nach ihrer Auffassung den Modus prägenden, aus dem gregorianischen Choral stammenden Formeln am deutlichsten

13 Tinctoris 1975, 75, zitiert nach Opitz (175).

14 Ebd., zitiert nach Opitz (221).

15 Ebd., 98, zitiert nach Opitz (304). zutage treten. Die ausgewählte Chanson wird dann sehr detailliert hinsichtlich ihres Modus mit Stücken aus dem weltlichen und aus dem geistlichen Repertoire sowohl von Binchois als auch von anderen Komponisten verglichen. Hierbei treten Quarten- und Quintenspezies als modusbestimmende Merkmale zugunsten melodischer Formeln deutlich in den Hintergrund. Am greifbarsten wird diese Tatsache im Fall der Chansons, die Opitz dem tetrardus zuschreibt (genauer gesagt seiner plagalen Form, da ein eigenständiger authentischer tetrardus bei Binchois nicht festzustellen sei). Die laut spseudo-klassischer Theorie für den tetrardus konstitutiven Spezies sind hier nämlich in den meisten Fällen kaum vorherrschend. Die b-Vorzeichen von tenor und contratenor verwandeln die Quintenspezies IV in die Quintenspezies I, denn der Halbtonschritt wird durch das $b$ von der dritten an die zweite Position des Skalenausschnitts $g-d$ versetzt. Hier wird nun Opitz' Verfahren, jeder Chanson einen eindeutigen Modus zuzuweisen, besonders problematisch. Denn das aufsteigende Quartintervall ist zwar, wie Opitz richtig bemerkt, für den plagalen tetrardus von ausschlaggebender Bedeutung. Es ist aber kaum zu leugnen, dass das Hervortreten von verschiedenen Spezies die Klangfülle des Satzes potentiell derartig färbt, dass man kaum noch von einem vorherrschenden Modus sprechen kann, auch wenn die im Choral 
für einen bestimmten Modus typischen Melodieformeln hie und da das polyphone Gewebe durchziehen. In dieser Hinsicht erfasst der von Tinctoris eingeführte Begriff der commixtio tonorum oder der Koexistenz von verschiedenen modusspezifischen Spezies adäquat die klangliche Wirklichkeit des Phänomens, und das umso mehr, als er gerade für die Mehrstimmigkeit eine große Bedeutung hat. Und schließlich scheint die Spezieslehre nichts anderes zu sein als der Versuch, die den verschiedenen gregorianischen Melodieformeln innewohnende klangliche ,Qualität herauszukristallisieren, eine `Qualitätı, die großenteils von der Position des Halbtonschritts innerhalb der konstitutiven Intervalle abhängt. Daher vermögen die Spezies eine Art komprimierter 'Quintessenz` der jeweiligen Melodieformel darzustellen, sodass ihr Auftauchen innerhalb einer Melodie auch die entsprechende Modalität - wenngleich nur lokal begrenzt - hervorruft. Aus diesem Grund laufen die großflächigen modalen Zuweisungen der Autorin Gefahr, die Schattierungen

\section{Literatur}

Allaire, Gaston G. (1972), The Theory of Hexachords, Solmization and the Modal System. A Practical Application, o. O.: American Institute of Musicology.

Berger, Christian (1992), Hexachord, Mensur und Textstruktur. Studien zum französischen Lied des 14. Jahrhunderts, Stuttgart: Steiner.

Boone, Graeme M. (1997), „Tonal Color in Dufay", in: Music in Renaissance Cities and Courts. Studies in Honor of Lewis Lockwood, hg. von Jessie Ann Owens und Anthony M. Cummings, Warren (MI): Harmonie Park Press, 57-99.

Bosi, Carlo (2007), »Analisi modale, polifonia e teoria musicale tardo-medievale. Un approccio storico-critico«, Rivista Italiana di Musicologia 42/1, 3-39. aus dem Auge zu verlieren, die das tatsächliche Vorhandensein verschiedener Spezies dem Satz verleihen.

Nichtsdestoweniger ist das Verfahren von Astrid Opitz, sich bei der modalen Analyse der Chansons Binchois' direkt an die Formeln der Gregorianik anzulehnen (ein Verfahren, das auch auf andere spätmittelalterliche Komponisten gewinnbringend angewandt werden kann), auf jeden Fall begrüßenswert, da es neue, auch praxisrelevante Perspektiven der theoretischen Auseinandersetzung mit der Mehrstimmigkeit dieser Zeit eröffnet. Daher trägt das Buch von Opitz wesentlich dazu bei, die musikalische Sprache spätmittelalterlicher Mehrstimmigkeit besser in ihrer Funktionsweise zu durchschauen und ist für jede*n Musikwissenschaftler*in mit Forschungsschwerpunkt auf Spätmittelalter und früher Renaissance und mit grundsätzlichen musiktheoretischen Vorkenntnissen eine höchst empfehlenswerte Lektüre.

\section{Carlo Bosi}

_ (2013), Emergence of Modality in Late Medieval Song. The Cases of Du Fay and Binchois, Würzburg: Königshausen \& Neumann.

Brothers, Thomas D. (1991), Genre, Style and Compositional Technique in French Music of the Fifteenth Century, Ph. D., University of California, Berkeley.

Fuller, Sarah (1998), »Modal Discourse and Fourteenth-Century French Song: A sMedievalı Perspective Recovered?", Early Music History 17, 61-108.

Marchettus von Padua (1987), Lucidarium in arte musicae planae. The Lucidarium of Marchetto of Padua. A Critical Edition, Translation, and Commentary, hg. von Jan W. Herlinger, Chicago (IL): University of Chicago Press. 
Meier, Bernhard (1953), »Die Handschrift Porto 714 als Quelle zur Tonartenlehre des 15. Jahrhunderts", Musica Disciplina 7, 175-197.

Perkins, Leeman L. (1996), »Modal Species and Mixtures in a Fifteenth-Century Chanson Repertory", in: Modality in the Music of the Fourteenth and Fifteenth Centuries / Modalität in der Musik des 14. und 15. Jahrhunderts, hg. von Ursula Günther, Ludwig Finscher und Jeffrey J. Dean, Neuhausen-Stuttgart: Hänssler, 177-201.

Powers, Harold S. (1992), »Is Mode Real? Pietro Aron, the Octenary System, and Polyphony", Basler Jahrbuch für historische Musikpraxis 16, 9-52.

Slavin, Dennis (2000), »The Binchois Game. Style and Tonal Coherence in Some Songs from the Mid-Fifteenth Century", in: Binchois Studies, hg. von Andrew Kirkman und Dennis Slavin, Oxford: Oxford University Press, 163-180.
Strohm, Reinhard (1996), »Modal Sounds as a Stylistic Tendency of the Mid-Fifteenth Century. E-, A-, and C-Finals in Polyphonic Song", in: Modality in the Music of the Fourteenth and Fifteenth Centuries / Modalität in der Musik des 14. und 15. Jahrhunderts, hg. von Ursula Günther, Ludwig Finscher und Jeffrey J. Dean, NeuhausenStuttgart: Hänssler, 149-175.

Tinctoris, Johannes (1975), Liber de natura et proprietate tonorum, in: Johannes Tinctoris: Theoretical Works, Bd. 1, hg. von Albert Seay, o. O.: American Institute of Musicology, 65-104.

Treitler, Leo (1965), »Tone System in the Secular Works of Guillaume Dufay", Journal of the American Musicological Society 18/2, 131-169. 